\title{
Dirac eigenvalues and total scalar curvature
}

\author{
Bernd Ammann and Christian Bär
}

23. June 1999

\begin{abstract}
It has recently been conjectured that the eigenvalues $\lambda$ of the Dirac operator on a closed Riemannian spin manifold $M$ of dimension $n \geq 3$ can be estimated from below by the total scalar curvature:

$$
\lambda^{2} \geq \frac{n}{4(n-1)} \cdot \frac{\int_{M} S}{\operatorname{vol}(M)} .
$$

We show by example that such an estimate is impossible.

1991 Mathematics Subject Classification: 58G25

Keywords: eigenvalues of the Dirac operator, total scalar curvature, Pinocchio metric
\end{abstract}

\section{Introduction}

Let $M$ be a closed Riemannian spin manifold of dimension $n \geq 2$. Then the eigenvalues of the Dirac operator $D$ acting on spinors form a discrete subset of $\mathbb{R}$ with finite multiplicities. Let $S: M \rightarrow \mathbb{R}$ denote the scalar curvature of $M$. Let $\nabla$ be the Levi-Civita connection on spinors. From the Schrödinger-Lichnerowicz formula [10, 9]

$$
D^{2}=\nabla^{*} \nabla+\frac{S}{4}
$$

it is clear that all eigenvalues $\lambda$ of $D$ must satisfy

$$
\lambda^{2} \geq \frac{1}{4} \min _{x \in M} S(x)
$$


because $\nabla^{*} \nabla$ is a nonnegative operator. Of course, this is interesting only if the scalar curvature is positive but then the estimate (2) is never sharp.

The first sharp estimate was given by T. Friedrich who proved

Friedrich inequality [5]. Let $M$ be a closed Riemannian spin manifold of dimension $n \geq 2$. Then all eigenvalues $\lambda$ of the Dirac operator D satisfy

$$
\lambda^{2} \geq \frac{n}{4(n-1)} \min _{x \in M} S(x) .
$$

This estimate is sharp in the sense that there are manifolds of positive scalar curvature where equality is attained for the eigenvalue of smallest modulus. The round sphere is the simplest such example. Friedrich's proof is based on a modification of the Levi-Civita connection and a formula similar to (1).

Still, the Friedrich inequality is nontrivial only if the scalar curvature is strictly positive. To allow at least some negative scalar curvature it can be improved in two ways. The first one works if the dimension is $n \geq 3$.

Hijazi inequality [7]. Let $M$ be a closed Riemannian spin manifold of dimension $n \geq 3$. Then all eigenvalues $\lambda$ of the Dirac operator D satisfy

$$
\lambda^{2} \geq \frac{n}{4(n-1)} \mu_{1}
$$

where $\mu_{1}$ is the first eigenvalue of the Yamabe operator $Y=4 \cdot \frac{n-1}{n-2} \cdot \Delta+S$.

Since $\Delta$ is a nonnegative operator the Hijazi inequality implies the Friedrich inequality if $n \geq 3$. Hijazi's proof uses Friedrich's modification of the LeviCivita connection and a clever conformal change of the metric. In dimension $n=2$ one can use a more complicated modification of the Levi-Civita connection and the Gauss-Bonnet theorem to show

Bär inequality [1], 2]. Let $M$ be a closed and connected Riemannian spin manifold of dimension $n=2$. Then all eigenvalues $\lambda$ of the Dirac operator $D$ satisfy

$$
\lambda^{2} \geq \frac{2 \pi \cdot \chi(M)}{\operatorname{area}(M)}
$$

where $\chi(M)$ is the Euler number of $M$. 
The estimate is nontrivial only if the surface is of genus 0 but this does not come as a surprise because surfaces of genus $\geq 1$ are know to have the Dirac eigenvalue 0 for suitable choice of spin structure and Riemannian metric.

It is now tempting to search for an improvement of the Hijazi inequality and to conjecture the following

Conjecture [6]. Let $M$ be a closed Riemannian spin manifold of dimension $n \geq 2$. Then all eigenvalues $\lambda$ of the Dirac operator D satisfy

$$
\lambda^{2} \geq \frac{n}{4(n-1)} \frac{\int_{M} S}{\operatorname{vol}(M)} .
$$

In dimension 2 this is exactly the Bär inequality. Unfortunately, the conjecture is false for $n \geq 3$ as we shall see. We will prove

Theorem. Let $M$ be a closed spin manifold of dimension $n \geq 3$. Then there exist constants $0<C_{1} \leq C_{2} \leq \ldots$ and a set $\mathcal{P}$ of Riemannian metrics on $M$ such that

- The $k^{\text {th }}$ Dirac eigenvalue $\lambda_{k}(g)$ (ordered by magnitude of its modulus) for the Riemannian metric $g$ is bounded by $C_{k}$,

$$
\lambda_{k}(g)^{2} \leq C_{k}
$$

for all $g \in \mathcal{P}$.

- The normalized total scalar curvature for the Riemannian metric $g$ is unbounded from above,

$$
\sup _{g \in \mathcal{P}} \frac{\int_{M} S_{g}}{\operatorname{vol}_{g}(M)}=\infty .
$$

Therefore an estimate of the type

$$
\lambda_{k}^{2} \geq C(k, n) \cdot \frac{\int_{M} S}{\operatorname{vol}(M)}
$$

is impossible in dimension $n \geq 3$. 


\section{Pinocchio Metrics}

In this section we will prove the theorem by explicitly constructing the set of metrics $\mathcal{P}$ with the desired properties. Let $M$ be a closed differentiable manifold of dimension $n \geq 3$ and a fixed spin structure. We choose a Riemannian metric $g_{0}$ on $M$ such that $(M, g)$ contains an embedded Euclidean ball $B$ of radius 1 .

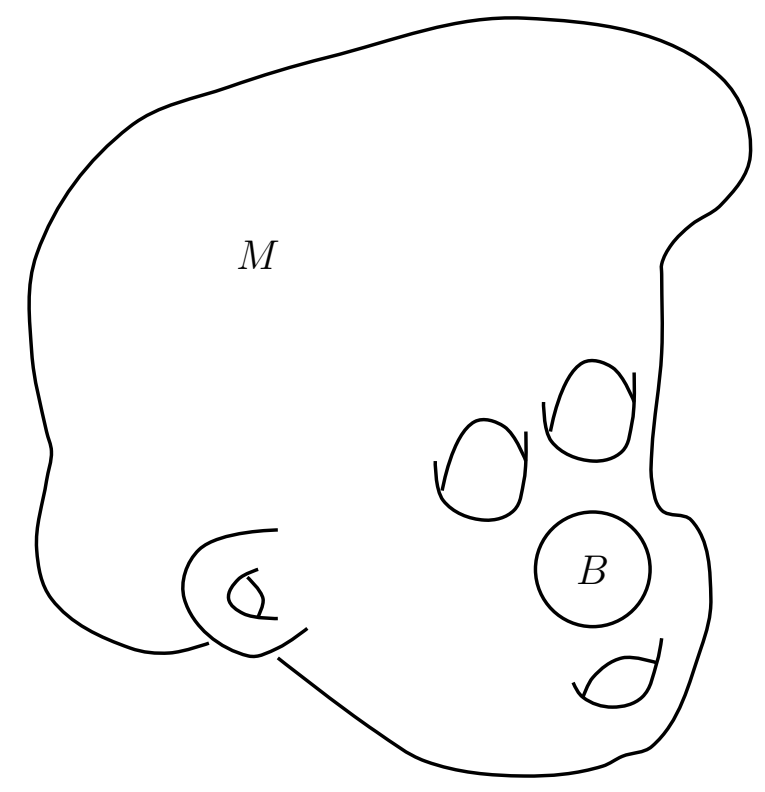

Fig. 1

Write the Euclidean ball $B$ as a union of two annuli and one smaller ball, $B=A_{1} \cup A_{2} \cup A_{3}$, where $A_{1}=\left\{x \in \mathbb{R}^{n}|2 / 3 \leq| x \mid \leq 1\right\}, A_{2}=\{x \in$ $\left.\mathbb{R}^{n}|1 / 3 \leq| x \mid \leq 2 / 3\right\}$ and $A_{3}=\left\{x \in \mathbb{R}^{n}|| x \mid \leq 1 / 3\right\}$. Now fix two parameters $0<r<1$ and $L>0$. Choose a Riemannian metric $g_{r, L}$ on $M$ with the following properties:

- $g_{r, L}$ coincides with $g_{0}$ on $M-B$ 
- $g_{r, L}$ is independent of $L$ on $A_{1}$ and on $A_{3}$

- $\left(A_{2}, g_{r, L}\right)$ is isometric to $S^{n-1}(r) \times[0, L]$ with the product metric where $S^{n-1}(r)$ denotes the round sphere of constant sectional curvature $1 / r^{2}$.

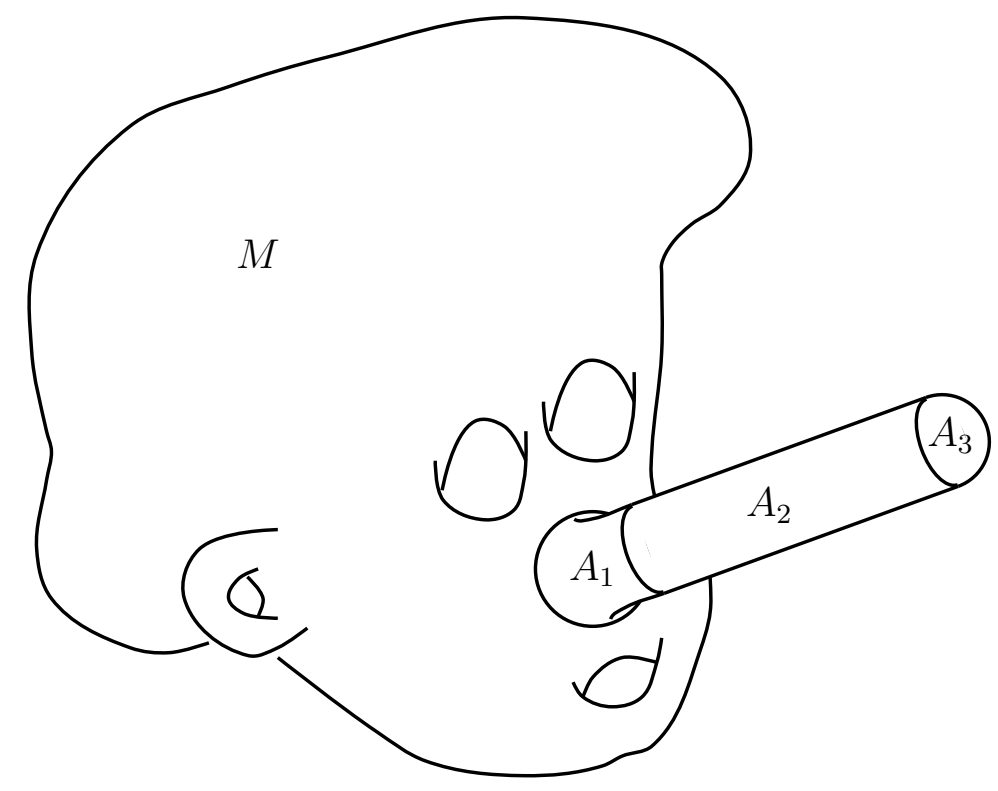

Fig. 2

Heuristically, there is a nose of radius $r$ and length $L$ growing out of the ball. For this reason we call these metrics Pinocchio metrics》. We set $\mathcal{P}:=$ $\left\{g_{r, L} \mid 0<r<1, L>0\right\}$ and we check the properties required in the theorem.

Claim 1. The $k^{\text {th }}$ eigenvalue $\lambda_{k}(r, L)^{2}$ of the square $D_{g_{r, L}}^{2}$ of the Dirac operator (w.r.t. the metric $g_{r, L}$ ) is bounded from above by a constant $C_{k}>0$ independent of $r$ and $L$.

\footnotetext{
${ }^{1}$ We are indebted to A. Hornecker for suggesting this name. There was another suggestion by L. Seeger to call them Viagra metrics which we rejected with regard to our readers under age.
} 
The proof is very simple. Choose a $k$-dimensional vector space $V_{k}$ of spinors $\psi$ on $M$ vanishing on $B$. Then $\psi \in V_{k}$ can be considered a spinor for all metrics $g_{r, L}$. We plug it into the Rayleigh quotient for the Dirac operator to get

$$
\begin{aligned}
\lambda_{k}(r, L)^{2} & \leq \sup _{\psi \in V_{k}, \psi \neq 0} \frac{\int_{M}\left\langle D_{g_{r, L}}^{2} \psi, \psi\right\rangle_{g_{r, L}}}{\int_{M}\langle\psi, \psi\rangle_{g_{r, L}}} \\
& =\sup _{\psi \in V_{k}, \psi \neq 0} \frac{\int_{M}\left\langle D_{g_{0}}^{2} \psi, \psi\right\rangle_{g_{0}}}{\int_{M}\langle\psi, \psi\rangle_{g_{0}}} \\
& =: \quad C_{k} .
\end{aligned}
$$

Claim 2. The normalized total scalar curvature is unbounded from above for $r \in(0,1)$ and $L \in(0, \infty)$.

Let $\omega_{k}$ denote the volume of the $k$-dimensional unit sphere. We compute

$$
\begin{aligned}
\frac{\int_{M} S_{g_{r, L}}}{\operatorname{vol}_{g_{r, L}}(M)} & =\frac{\int_{(M-B) \cup A_{1} \cup A_{3}} S_{g_{r, L}}+\int_{A_{2}} S_{g_{r, L}}}{\operatorname{vol}_{g_{r, L}}\left((M-B) \cup A_{1} \cup A_{3}\right)+\operatorname{vol}_{g_{r, L}}\left(A_{2}\right)} \\
& =\frac{\int_{(M-B) \cup A_{1} \cup A_{3}} S_{g_{r, L}}+L \cdot \frac{(n-1)(n-2)}{r^{2}} \cdot r^{n-1} \cdot \omega_{n-1}}{\operatorname{vol}_{g_{r, L}}\left((M-B) \cup A_{1} \cup A_{3}\right)+L \cdot r^{n-1} \cdot \omega_{n-1}} \\
& \longrightarrow \frac{(n-1)(n-2)}{r^{2}}
\end{aligned}
$$

for $L \rightarrow \infty$ because on $M-A_{2}$ the metric $g_{r, L}$ does not depend on $L$ by construction. Hence

$$
\sup _{L>0} \frac{\int_{M} S_{g_{r, L}}}{\operatorname{vol}_{g_{r, L}}(M)} \geq \frac{(n-1)(n-2)}{r^{2}}
$$

and therefore

$$
\sup _{L>0,0<r<1} \frac{\int_{M} S_{g_{r, L}}}{\operatorname{vol}_{g_{r, L}}(M)}=\infty .
$$

This proves the theorem.

\section{Concluding Remarks}

There is another reason why conjecture (3) cannot be true. Assume that the closed spin manifold $M$ of dimension $n \geq 3$ has a metric $g$ with harmonic 
spinors. It is known that such a metric always exists if $n \equiv 0,1,3,7$ modulo 8 [8, 3]. If the conjecture were true, then the total scalar curvature of every conformally equivalent metric $g_{1}$ would have to be nonpositive because the multiplicity of the Dirac eigenvalue 0 is a conformal invariant. But it is wellknown that the total scalar curvature functional is not bounded from above on any conformal class. Here is the simple argument. Write $g_{1}=u^{4 /(n-2)} \cdot g$ for some positive function $u$. Then the scalar curvature of $g_{1}$ is given by

$$
S_{1}=u^{-\frac{n+2}{n-2}} \cdot Y(u)
$$

where $Y$ is the Yamabe operator as defined in the Hijazi inequality. The volume element is

$$
d v o l_{1}=u^{\frac{2 n}{n-2}} d v o l
$$

and thus the total scalar curvature is

$$
\int_{M} S_{1} d v o l_{1}=\int_{M} u \cdot Y(u) d v o l
$$

cf. [4, Ch. 1.J]. Pick an eigenfunction $u$ of $Y$ for some positive eigenvalue $\mu>0$. The function $u$ will not be positive but for $\epsilon>0$ we can define $u_{\epsilon}:=\sqrt{u^{2}+\epsilon}$ and we see easily that

$$
\int_{M} u_{\epsilon} \cdot Y\left(u_{\epsilon}\right) d v o l \stackrel{\epsilon \unlhd 0}{\longrightarrow} \int_{M} u \cdot Y(u) d v o l=\mu \cdot \int_{M} u^{2} d v o l
$$

Thus the total scalar curvature functional is not bounded from above on the conformal class of $g$.

For the proof of Claim 1 the use of the Dirac operator $D$ was rather inessential. What we used is this: The operator $D^{2}$ is elliptic and self-adjoint, it is computed locally out of the metric and its derivatives, and its eigenvalues can be characterized by variation of the Rayleigh quotient. The theorem will still hold with $D^{2}$ replaced by any other operator having these properties. For example, we can take the Laplace operator acting on differential forms. If we denote the $k^{\text {th }}$ eigenvalue of the Laplace operator (w.r.t. the Riemannian metric $g$ ) acting on $p$-forms by $\lambda_{k}^{p}(g)$, then we see that

$$
\sup _{g \in \mathcal{P}} \lambda_{k}^{p}(g)<\infty
$$


Hence an estimate of the type

$$
\lambda_{k}^{p} \geq C(k, n) \cdot \frac{\int_{M} S}{\operatorname{vol}(M)}
$$

is also impossible in dimension $n \geq 3$.

\section{References}

[1] C. Bär, Das Spektrum von Dirac-Operatoren, Doktorarbeit, Universität Bonn, 1991, Bonner Math. Schr. 217.

[2] _ Lower eigenvalue estimates for Dirac operators, Math. Ann. 293 (1992), 39-46.

[3] _ Metrics with harmonic spinors, Geom. Func. Anal. 6 (1996), 899-942.

[4] A. L. Besse, Einstein manifolds, Springer-Verlag, Berlin-Heidelberg, 1987.

[5] T. Friedrich, Der erste Eigenwert des Dirac-Operators einer kompakten Riemannschen Mannigfaltigkeit nicht-negativer Krümmung, Math. Nachr. 97 (1980), 117-146.

[6] T. Friedrich and E.C. Kim, Some remarks on the Hijazi inequality and generalizations for the Killing equation for spinors, Preprint, prelim. version (1999).

[7] O. Hijazi, A conformal lower bound for the smallest eigenvalue of the Dirac operator and Killing spinors, Comm. Math. Phys. 104 (1986), 151-162.

[8] N. Hitchin, Harmonic spinors, Adv. Math. 14 (1974), 1-55.

[9] A. Lichnerowicz, Spineurs harmoniques, C. R. Acad. Sci. Paris 257 (1963), 7-9.

[10] E. Schrödinger, Diracsches Elektron im Schwerefeld I., Sitzungsber. Preuss. Akad. Wiss., Phys.-Math. Kl. (1932), 105-128. 
Mathematisches Institut

Universität Freiburg

Eckerstr. 1

79104 Freiburg

Germany

E-Mail: ammann@mathematik.uni-freiburg.de

baer@mathematik. uni-freiburg.de

WWW: http://web.mathematik.uni-freiburg.de/home/ammann

http://web.mathematik.uni-freiburg.de/home/baer 